\title{
Neutron cross section covariances in the resolved resonance region
}

\author{
M. Herman, S.F. Mughabghab, P. Obložinský, M.T. Pigni \\ D. Rochman*
}

National Nuclear Data Center, Brookhaven National Laboratory

Upton, New York, 11973-5000, U.S.A.

www.nndc.bnl.gov

April 2008

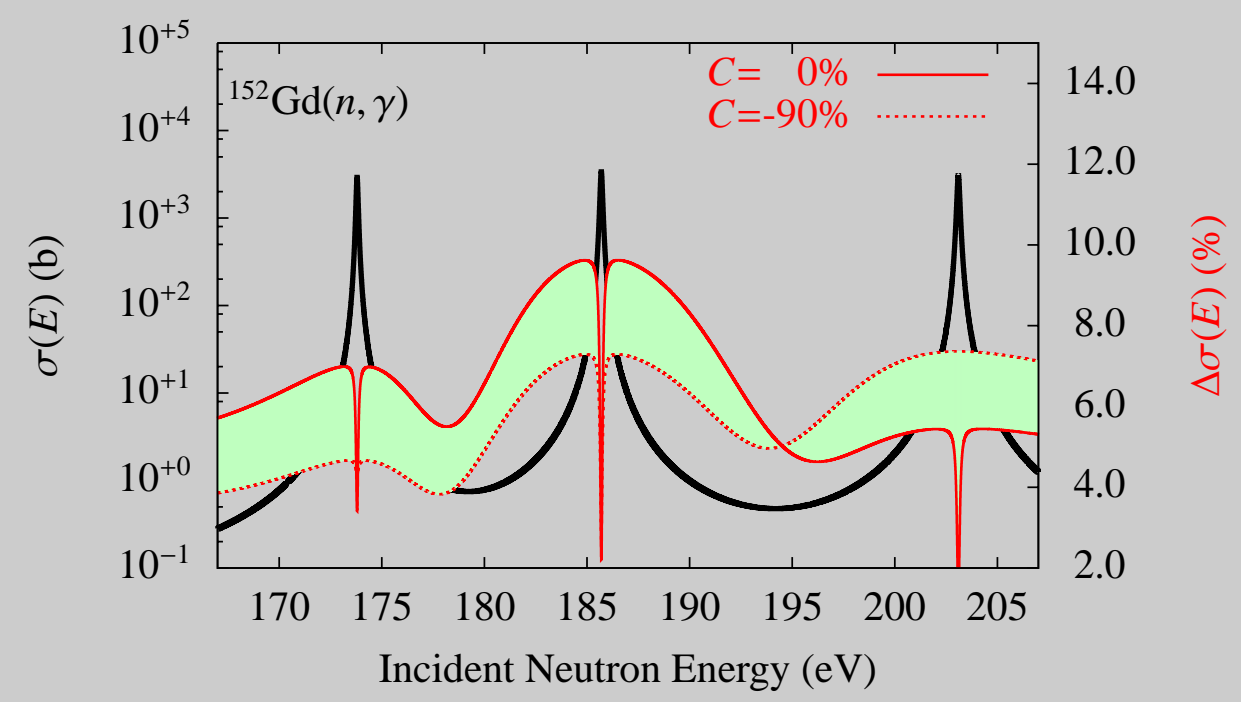

* Address since September 2007: Nuclear Research and Consultancy Group, NRG, P.O. Box 25, 1755 ZG Petten, The Netherlands.

Notice: This manuscript has been authored by employees of Brookhaven Science Associates, LLC under Contract No. DE-AC02-98CH10886 with the U.S. Department of Energy. The publisher by accepting the manuscript for publication acknowledges that the United States Government retains a non-exclusive, paid-up, irrevocable, world-wide license to publish or reproduce the published form of this manuscript, or allow others to do so, for United States Government purposes. 


\section{DISCLAIMER}

This report was prepared as an account of work sponsored by an agency of the United States Government. Neither the United States Government nor any agency thereof, nor any of their employees, nor any of their contractors, subcontractors, or their employees, makes any warranty, express or implied, or assumes any legal liability or responsibility for the accuracy, completeness, or any third party's use or the results of such use of any information, apparatus, product, or process disclosed, or represents that its use would not infringe privately owned rights. Reference herein to any specific commercial product, process, or service by trade name, trademark, manufacturer, or otherwise, does not necessarily constitute or imply its endorsement, recommendation, or favoring by the United States Government or any agency thereof or its contractors or subcontractors. The views and opinions of authors expressed herein do not necessarily state or reflect those of the United States Government or any agency thereof. 


\section{Contents}

1 Introduction 1

2 Capture and fission cross sections 4

3 Cross section covariances for a single resonance 6

3.1 Cross section uncertainties . . . . . . . . . . . . . . . 6

3.2 Cross section correlations . . . . . . . . . . . . . . . 8

3.3 Averaged values . . . . . . . . . . . . . . 13

4 Cross section covariances for multiple resonances 16

4.1 Cross section uncertainties . . . . . . . . . . . . . 16

4.2 Cross section correlations . . . . . . . . . . . . . . . 20

4.3 Averaged values . . . . . . . . . . . . . . 22

5 Conclusions 23

List of Figures

List of Tables

$\begin{array}{ll}\text { Acknowledgements } & 27\end{array}$

Bibliography $\quad 29$ 



\begin{abstract}
We present a detailed analysis of the impact of resonance parameter uncertainties on covariances for neutron capture and fission cross sections in the resolved resonance region. Our analysis uses the uncertainties available in the recently published Atlas of Neutron Resonances employing the Multi-Level Breit-Wigner formalism. We consider uncertainties on resonance energies along with those on neutron-, radiative-, and fission-widths and examine their impact on cross section uncertainties and correlations. We also study the effect of the resonance parameter correlations deduced from capture and fission kernels and illustrate our approach on several practical examples. We show that uncertainties of neutron-, radiativeand fission-widths are important, while the uncertainties of resonance energies can be effectively neglected. We conclude that the correlations between neutron and radiative (fission) widths should be taken into account. The multi-group cross section uncertainties can be properly generated from both the resonance parameter covariance format MF32 and the cross section covariance format MF33, though the use of MF32 is more straightforward and hence preferable.
\end{abstract}

Editorial note: The ideas on which this paper is based were put forward during numerous discussions between the scientists of the National Nuclear Data Center, BNL in the first half of 2007. This was part of an intensive effort devoted to developing neutron cross section covariance methodology in the resolved resonance region. The backbone of this methodology is the use of the uncertainty information contained in the Atlas of Neutron Resonances (author S. Mughabghab, Elsevier 2006). The present report was drafted in summer 2007, near final version followed in September 2007. Three months later, in December 2007, a paper by D. Rochman and A.J. Koning, NRG Petten, was submitted to Nucl. Instr. Methods A using many of our original ideas without mentioning our work. The NNDC learned about it from an on-line version of NIM-A in March 2008. This prompted publishing the present report in order to secure our priority in this matter. 



\section{Chapter 1}

\section{Introduction}

The recent revival of interest in neutron cross section covariances (uncertainties and correlations) is driven by the needs of advanced reactor systems and fuel cycles [1,2], data adjustment for the Global Nuclear Energy Partnership (GNEP) project as well as nuclear criticality safety. This interest is strongly enhanced by recent advances in computer technology and progress in radiation transport codes allowing to perform fast numerical simulations. Such simulations can substantially reduce expensive and time consuming measurements on mock-up assemblies. For these simulations to be useful, neutron cross section evaluations have to come with a trusted estimate of uncertainties.

It appears that the covariance information is very incomplete even in the most recent nuclear data libraries. For example, the brand new ENDF/B-VII.0 library [3] contains neutron cross section covariances only for 13 old and 13 newly evaluated materials out of 393. The consequence of the lack of covariance information in the user community is a common misuse assuming that a given old covariance file, obtained under specific conditions, for specific cross sections or other nuclear data, can be used with a new data file, obtained under different assumptions. To remedy this problem, it is important to create new reliable covariance files, consistent with mean values to which they refer to.

The new neutron cross section covariances included in the ENDF/B-VII.0 library are sample covariance evaluations that represent a prerequisite for a much broader effort anticipated for ENDF/B-VII.1 release. In the resolved resonance region these evaluations were obtained by three different methods. The direct SAMMY was used for the covariance evaluation of ${ }^{232} \mathrm{Th}$, the retroactive SAMMY for ${ }^{152,153,154,155,156,157,158,160} \mathrm{Gd}$, and the Atlas-KALMAN method was used for evaluation of ${ }^{89} \mathrm{Y},{ }^{99} \mathrm{Tc}$ and ${ }^{191,193} \mathrm{Ir}$. 
The first method, direct SAMMY, is the most suitable for new measurements, where the analysis of raw experimental data can be performed with powerful Rmatrix codes. The best known is the ORNL code SAMMY [4], which automatically produces full covariance information [5]. For comparison, the European code REFIT [6] has similar capabilities in data analysis [7], but produces diagonal covariance terms only. The code SAMMY preforms a multilevel multichannel R-matrix fit to neutron data using the Reich-Moore formalism. Experimental conditions such as resolution function, finite size sample, non-uniform thickness of sample, multiple scattering, self-shielding, normalization, background are taken into account. An important distinction of the SAMMY is the usage of the Bayes' equations, or the generalized least squares rather than the least-squares equations to update resonance parameters. The difference, making SAMMY more powerful, lies in the assumption implicit in the least squares that the prior parameter covariance matrix is infinite and diagonal [8].

The second method is based on the idea to generate experimental data "retroactively" and then proceed with the direct evaluation as described above [9]. The motivation behind this somewhat unorthodox method, termed retroactive SAMMY [3], is to benefit from the power of SAMMY and from huge experience accumulated over years in experimental facilities such as ORELA. An intention is to apply this method to those cases where suitable experimental data are not available. In doing so one first generates artificial experimental cross sections using the R-matrix theory with already-determined values of resonance parameters. Statistical and systematical uncertainties are assigned to each data point, estimated from past experience. Transmission, capture, fission and other data are calculated assuming realistic experimental conditions such as Doppler broadening and resolution function. Then, the SAMMY code is used to generate resonance-parameter covariance matrix.

The third method, pursued by the National Nuclear Data Center, is focusing on many cases where the use of the above two methods may not be practical. It is based on the idea to utilize another resource of information on neutron resonances, namely, the recently published Atlas of Neutron Resonances [10]. This monumental work by S.F. Mughabghab represents the $5^{\text {th }}$ edition of what was previously well known as the Brookhaven National Laboratory BNL-325 Reports. The point is that Atlas contains not only the resonance parameters, frequently adopted by many evaluations in major evaluated data libraries, but also their uncertainties. The idea is to make use of these uncertainties and convert them into neutron cross section covariances. Such a task has several distinct perspectives. 
- One perspective is that we deal with a specific case of nuclear reaction modeling that one would ideally encounter when using the nuclear reaction model code EMPIRE originally designed for evaluations in the fast neutron region [11]. In EMPIRE, one is far away from a situation of having perfect model, perfect parametrization along with solid model parameter uncertainties. Yet, the resolved resonance region is pretty close to this ideal situation. One has a model, such as the Multi-Level Breit-Wigner (MLBW) formalism, with a set of well determined model parameters along with their uncertainties directly deduced from experiments. Hence, one should built on experience from coupling EMPIRE with the Bayesian code KALMAN [12] to produce covariances in the fast neutron region and expand it to the resonance region. This led to the development of the Atlas-KALMAN method, used to evaluate four materials for ENDF/B-VII.0 [3] and also to produce preliminary set of covariances for advanced reactor systems [13].

- Another perspective is that one encounters a typical processing problem, with converting resonance parameters (file MF2 as defined in the ENDF6 format [14]) and the resonance parameter uncertainties (file MF32) into cross sections and cross section covariances. To this end, one should employ a suitable processing code such as PUFF [15] or ERRORJ [16]. This approach, however tempting, does not provide sufficient insight into the role of the resonance parameter uncertainties unless one is sufficiently familiar with the processing code itself.

- Still another perspective is that one deals with the task where straightforward analytical solutions are possible. This should shed sufficient light on the role of the resonance parameter uncertainties and this is the primary objective of the present paper. On practical level, such an analysis would bring us to the previous item by providing justification for conversion of uncertainty information from the Atlas of Neutron Resonances into MF32 covariances. This procedure is straightforward and should be preferred over our earlier approach of using MF33.

This paper is organized as follows. In Chapter 2 we summarize formalism for neutron capture and fission cross sections. In Chapter 3 we consider single resonances and analyze the impact of the resonance parameter uncertainties and resonance parameter correlations on the neutron cross section uncertainties and correlations. Then, in Chapter 4 we extend this analysis to many resonances. Our conclusions are given in Chapter 5. 


\section{Chapter 2}

\section{Capture and fission cross sections}

We restrict ourselves to the MLBW formalism as defined in the ENDF-6 format [14]. This is justified by a wide use of MLBW in all major evaluated nuclear data libraries and its dominant use also in the Atlas of Neutron Resonances. Furthermore, MLBW is sufficiently representative for our purposes and relatively easy to implement analytically. Although our analysis could be extended to a more sophisticated Reich-Moore formalism, it would hardly change any of our findings.

For a simplicity we restrict ourselves to s-wave processes, first discuss a single resonance, then proceed with a multi-resonance case. We will provide expressions for capture cross sections, with the understanding that the expressions for fission cross sections can be obtained by a simple transformation. For the purposes of the present paper all examples shown to illustrate our points are s-wave resonances.

For a single resonance at the energy $E_{0}$ and the neutron incident energy $E$, the capture cross section can be expressed by the Breit-Wigner formula as

$$
\sigma_{\gamma}(E)=\pi \lambda^{2} \frac{g \Gamma_{n}(E) \Gamma_{\gamma}}{(\Gamma(E) / 2)^{2}+\left(E-E_{0}\right)^{2}},
$$

where we dropped all indices related to quantum numbers. Here, $t$ is the neutron wavelength,

$$
\lambda=\frac{\hbar}{\sqrt{2 m E}},
$$

$m$ being the neutron reduced mass and $\hbar$ the Planck constant, the spin statistical factor is given by

$$
g=\frac{2 J+1}{2(2 I+1)}
$$


with $J$ being the spin of the resonance and $I$ the spin of the target nucleus, and the energy-dependent neutron width for s-wave neutrons is

$$
\Gamma_{n}(E)=\Gamma_{n} \sqrt{\frac{E}{E_{0}}},
$$

where $\Gamma_{n}$ denotes the neutron width at $E_{0}$. The energy dependence of the total resonance width, $\Gamma(E)$, can be neglected when compared to the strong energy term in the denominator of Eq. 2.1, giving

$$
\Gamma=\Gamma_{n}+\Gamma_{\gamma}+\Gamma_{f}
$$

being $\Gamma_{\gamma}$ and $\Gamma_{f}$ the radiative and fission width respectively. Eq. (2.1) can be rewritten to its final form

$$
\sigma_{\gamma}(E)=\frac{2 \pi \hbar^{2}}{m}\left(\frac{1}{E E_{0}}\right)^{1 / 2} \frac{g \Gamma_{n} \Gamma_{\gamma}}{\left(\Gamma_{n}+\Gamma_{\gamma}+\Gamma_{f}\right)^{2}+4\left(E-E_{0}\right)^{2}},
$$

where one can explicitly see all quantities of interest to our analysis. These quantities, along with their uncertainties, can in general be found in the Atlas of Neutron Resonances [10] and include the resonance parameters $E_{0}, \Gamma_{n}, \Gamma_{\gamma}, \Gamma_{f}$ and the capture kernel $g \Gamma_{n} \Gamma_{\gamma} / \Gamma$.

For the case of several resonances the above expression can be generalized by performing summation over the individual resonances, denoted by the subscript $r$,

$$
\begin{aligned}
\sigma_{\gamma}(E) & =\sum_{r} \sigma_{\gamma r}(E) \\
& =\frac{2 \pi \hbar^{2}}{m} \sum_{r}\left(\frac{1}{E E_{0 r}}\right)^{1 / 2} \frac{g_{r} \Gamma_{n r} \Gamma_{\gamma r}}{\Gamma_{r}^{2}+4\left(E-E_{0 r}\right)^{2}} .
\end{aligned}
$$

This is justified by the observation that there are no interference effects in neutron capture, generally when the number of primary $\gamma$-ray transitions is large.

For fission cross sections the same formalism, after interchanging the subscripts $\gamma$ and $f$ in the above equations, can be applied. 


\section{Chapter 3}

\section{Cross section covariances for a single resonance}

The energy-energy covariance between capture cross sections, $\sigma_{\gamma}(E)$ and $\sigma_{\gamma}\left(E^{\prime}\right)$ at the neutron energies $E$ and $E^{\prime}$, is given by

$$
\left\langle\delta \sigma_{\gamma}(E) \delta \sigma_{\gamma}\left(E^{\prime}\right)\right\rangle=\sum_{i, j} \frac{\partial \sigma_{\gamma}(E)}{\partial p_{i}}\left\langle\delta p_{i} \delta p_{j}\right\rangle \frac{\partial \sigma_{\gamma}\left(E^{\prime}\right)}{\partial p_{j}},
$$

where $p_{i}$ stands for the resonance parameters $E_{0}, \Gamma_{n}, \Gamma_{\gamma}, \Gamma_{f}$, and $\left\langle\delta p_{i} \delta p_{j}\right\rangle$ is their covariance matrix. Assuming that the resonance parameters are uncorrelated,

$$
\left\langle\delta p_{i} \delta p_{j}\right\rangle=\left\{\begin{array}{cl}
\left(\Delta p_{i}\right)^{2} & i=j \\
0 & i \neq j
\end{array}\right.
$$

one gets

$$
\left\langle\delta \sigma_{\gamma}(E) \delta \sigma_{\gamma}\left(E^{\prime}\right)\right\rangle=\sum_{i} \frac{\partial \sigma_{\gamma}(E)}{\partial p_{i}}\left(\Delta p_{i}\right)^{2} \frac{\partial \sigma_{\gamma}\left(E^{\prime}\right)}{\partial p_{i}}
$$

that defines all elements of the energy-energy cross section covariance matrix. The diagonal terms, $E=E^{\prime}$, contain cross section uncertainties, while the off-diagonal terms, $E \neq E^{\prime}$, contain cross section correlations.

\subsection{Cross section uncertainties}

The diagonal terms of the energy-energy covariance matrix are cross section uncertainties. Using a more explicit notation, this diagonal term defined by Eq. (3.3) 
can be written for non-fissile nuclei as

$$
\left(\Delta \sigma_{\gamma}\right)^{2}=\left(\frac{\partial \sigma_{\gamma}}{\partial E_{0}} \Delta E_{0}\right)^{2}+\left(\frac{\partial \sigma_{\gamma}}{\partial \Gamma_{n}} \Delta \Gamma_{n}\right)^{2}+\left(\frac{\partial \sigma_{\gamma}}{\partial \Gamma_{\gamma}} \Delta \Gamma_{\gamma}\right)^{2}
$$

Here, $\partial \sigma_{\gamma} / \partial E_{0}, \partial \sigma_{\gamma} / \partial \Gamma_{n}$, and $\partial \sigma_{\gamma} / \partial \Gamma_{\gamma}$ are the partial derivatives and $\Delta E_{0}, \Delta \Gamma_{n}$, and $\Delta \Gamma_{\gamma}$ are the standard deviations (uncertainties) of the resonance energy, neutron, radiative width, respectively. We note that the above equation can be easily generalized to describe actinides by adding fission term.

Considering Eq. (2.6), the first term of Eq. (3.4), after normalizing it to the capture cross section, gives the relative capture cross section uncertainty

$$
\frac{\partial \sigma_{\gamma}}{\partial E_{0}} \frac{\Delta E_{0}}{\sigma_{\gamma}}=\left(\frac{8 E_{0}\left(E-E_{0}\right)}{\Gamma^{2}+4\left(E-E_{0}\right)^{2}}-\frac{1}{2}\right) \frac{\Delta E_{0}}{E_{0}},
$$

which shows strong $E$-dependence. Thus, for the neutron energies far away from $E_{0}$ the cross section uncertainty is small, -(5/2) $\Delta E_{0} / E_{0}$ at $E=0$ and -(1/2) $\Delta E_{0} / E_{0}$ at $E>>E_{0}$. For the interim energies, the leading term is $2 \Delta E_{0} /\left(E-E_{0}\right)$ and this explains the initial rapid growth in the relative cross section uncertainty, followed by equally rapid decrease, with a deep minimum at $E=E_{0}$.

As an example, in Fig. 3.1 we show ${ }^{152} \mathrm{Gd}(n, \gamma)$ for the single s-wave resonance with the resonance energy $E_{0}=173.8 \mathrm{eV}$ known to $0.06 \%$ precision, see Table 3.1, while $\Gamma$ and $\Gamma_{\gamma}$ are treated as exactly known quantities. Although the cross section

Table 3.1: The resonance parameters and their uncertainties for $E_{0}=173.8 \mathrm{eV} \mathrm{s}$-wave resonance in ${ }^{152} \mathrm{Gd}+n$ [10].

\begin{tabular}{ccc}
\hline $\mathrm{E}_{0}(\mathrm{eV})$ & $\mathrm{g} \Gamma_{n}(\mathrm{meV})$ & $\Gamma_{\gamma}(\mathrm{meV})$ \\
\hline $173.8 \pm 0.1$ & $86 \pm 2$ & $30 \pm 2$ \\
\hline
\end{tabular}

uncertainties tend to be very large, in practice they can be neglected since there is a strong anti-correlation with respect to $E_{0}$ (see Sec. 3.2). This anti-correlation virtually annihilates contribution to cross section uncertainties due to $\Delta E_{0}$ once the cross section averaging is done even over the fairly narrow energy interval around $E_{0}$.

The second term in Eq. (3.4), the energy dependence of the relative capture cross section uncertainty due to $\Delta \Gamma_{x}$, reads

$$
\frac{\partial \sigma_{\gamma}}{\partial \Gamma_{x}} \frac{\Delta \Gamma_{x}}{\sigma_{\gamma}}=\left(1-\frac{2 \Gamma_{x} \Gamma}{\Gamma^{2}+4\left(E-E_{0}\right)^{2}}\right) \frac{\Delta \Gamma_{x}}{\Gamma_{x}} .
$$




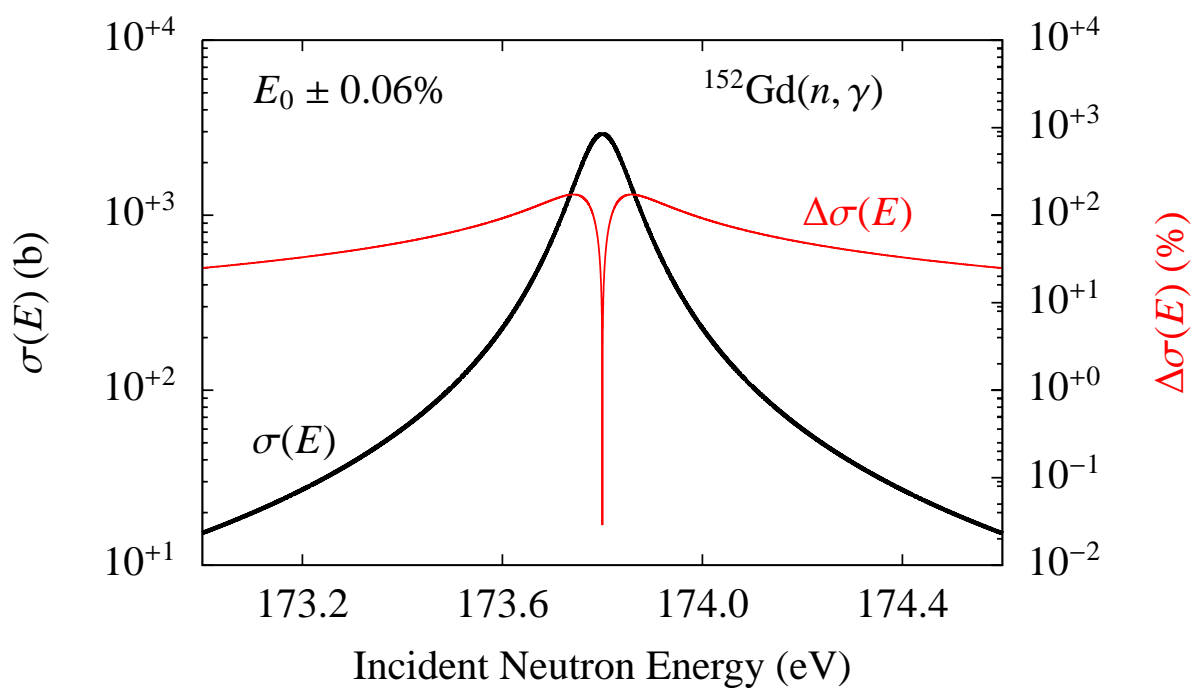

Figure 3.1: The ${ }^{152} \mathrm{Gd}(n, \gamma)$ cross sections for the single resonance $E_{0}=173.8 \mathrm{eV}$ (left scale) and their relative uncertainties due to the resonance energy uncertainty $0.06 \%$ (right scale).

where the index $x$ stands either for $n$ or $\gamma$. This expression gives the cross section uncertainties that are fairly constant. For the neutron energies far away from $E_{0}$ one gets $\Delta \Gamma_{x} / \Gamma_{x}$ for cross section uncertainty, the interim energy region is fairly flat, with somewhat complex shape close to $E_{0}$ depending on the actual value of the term $\left(1-2 \Gamma_{x} / \Gamma\right)$.

An example is given for ${ }^{152} \mathrm{Gd}(n, \gamma)$ for the single resonance $E_{0}=173.8 \mathrm{eV}$, with $\Delta \Gamma_{n} / \Gamma_{n}=2.3 \%$ and $\Delta \Gamma_{\gamma} / \Gamma_{\gamma}=6.6 \%$, see Table 3.1. Shown in Fig. 3.2 is the impact of $\Delta \Gamma_{n}$ which yields complex shape around $E_{0}$ caused by $\Gamma_{n} / \Gamma$ being close to unity. Fig. 3.3 shows the contribution caused by $\Delta \Gamma_{\gamma}$ that drops at $E_{0}$ since $\Gamma_{\gamma} / \Gamma$ is relatively small.

\subsection{Cross section correlations}

The correlation between capture cross sections is given by the non-diagonal terms, $E \neq E^{\prime}$, of the energy-energy covariance matrix, Eq. (3.3). Two possibilities will be discussed. First, we will consider the uncorrelated resonance parameters. Then, we will examine the correlation between $\Gamma_{n}$ and $\Gamma_{\gamma}$ using the constraint given by the capture kernel. 


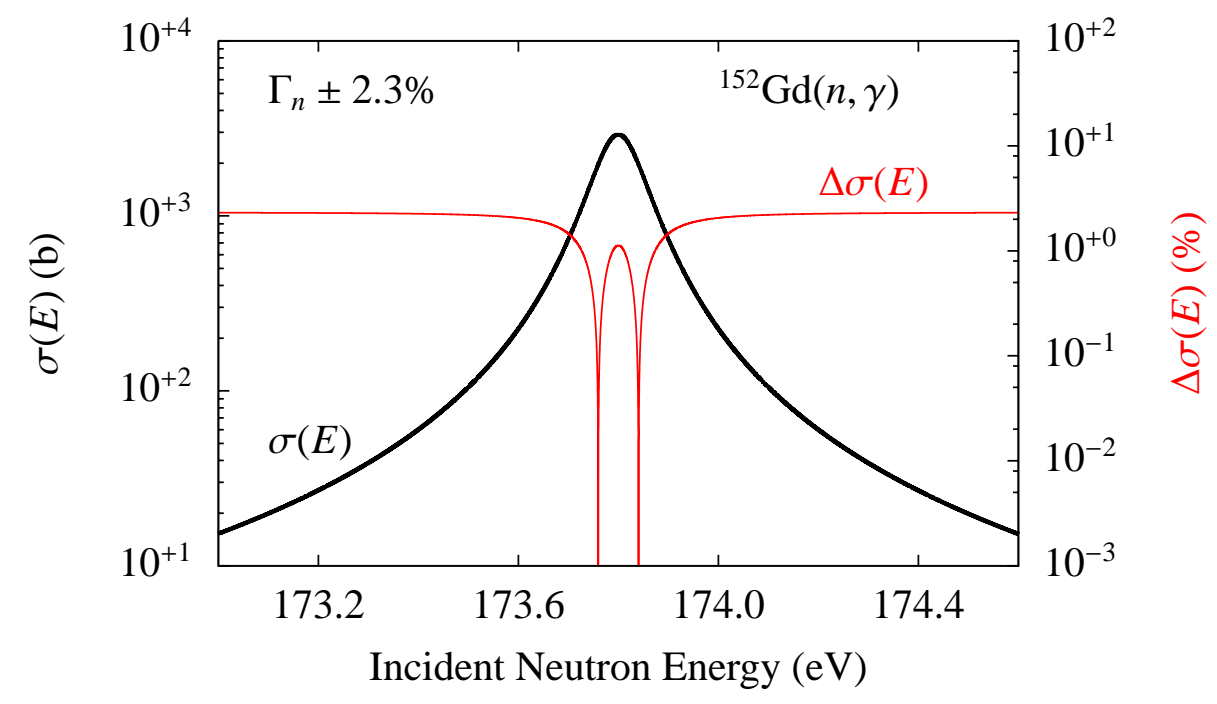

Figure 3.2: The ${ }^{152} \mathrm{Gd}(n, \gamma)$ cross sections for the single $173.8 \mathrm{eV}$ resonance (left scale) and their relative uncertainties due to the neutron width $\Gamma_{n}=86 \mathrm{meV} \pm 2.3 \%$ (right scale).

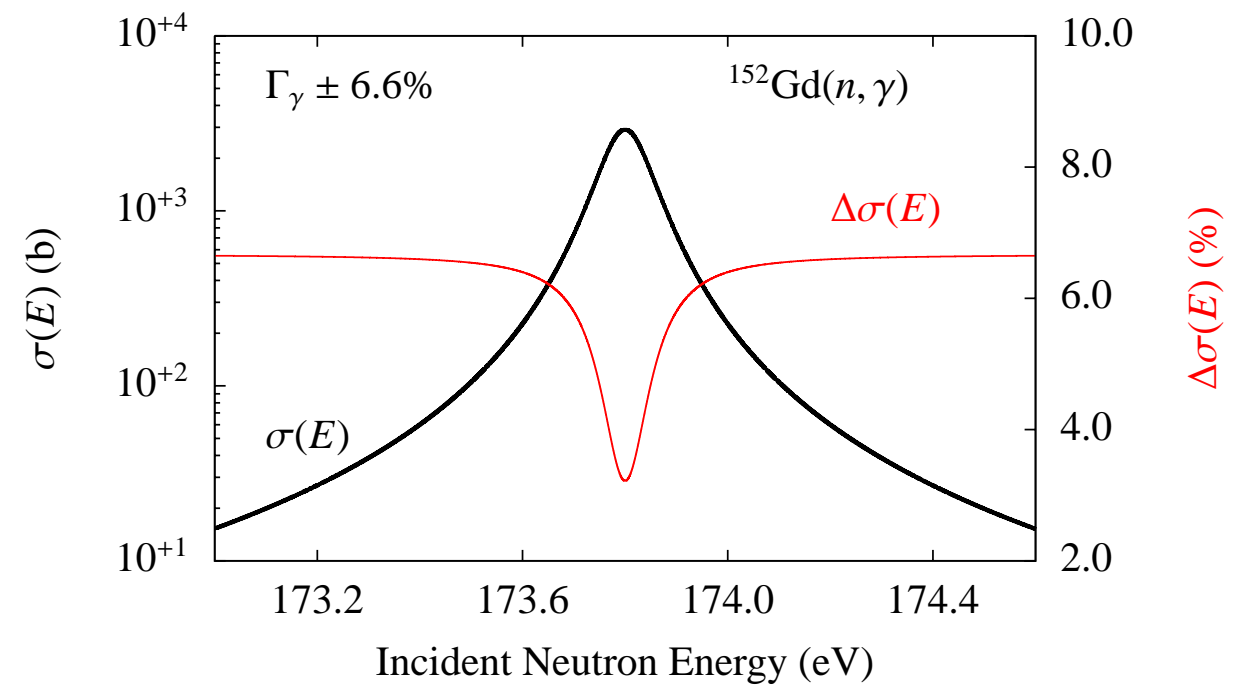

Figure 3.3: The ${ }^{152} \mathrm{Gd}(n, \gamma)$ cross sections for the single $173.8 \mathrm{eV}$ resonance (left scale) and their relative uncertainties due to the radiative width $\Gamma_{\gamma}=30 \mathrm{meV} \pm 6.6 \%$ (right scale). 
For the uncorrelated resonance parameters, and following the usual practice to normalize the covariance matrix so that the matrix elements are between -1 and +1 , one gets correlation matrix

$$
\frac{\left\langle\delta \sigma_{\gamma}(E) \delta \sigma_{\gamma}\left(E^{\prime}\right)\right\rangle}{\Delta \sigma_{\gamma}(E) \Delta \sigma_{\gamma}\left(E^{\prime}\right)}=\sum_{i} \frac{\partial \sigma_{\gamma}(E)}{\partial p_{i}} \frac{\left(\Delta p_{i}\right)^{2}}{\Delta \sigma_{\gamma}(E) \Delta \sigma_{\gamma}\left(E^{\prime}\right)} \frac{\partial \sigma_{\gamma}\left(E^{\prime}\right)}{\partial p_{i}}
$$

where $p_{i}=E_{0}, \Gamma_{n}, \Gamma_{\gamma}$. For illustration we continue to analyze ${ }^{152} \mathrm{Gd}(n, \gamma)$ at $E_{0}=173.8 \mathrm{eV}$. In Fig. 3.4, to the right, we show the relative cross section uncertainties due to both the neutron and radiative widths uncertainties, $\Delta \Gamma_{n}$ and $\Delta \Gamma_{\gamma}$, while the resonance energy $E_{0}$ is considered to be known exactly. Then, in Fig. 3.5 we show a complete case, where also the resonance energy uncertainty, $\Delta E_{0}$, is considered. This has striking impact, showing up as strong anti-correlation with respect to the energy $E_{0}$. As a consequence this anti-correlation annihilates the impact of $\Delta E_{0}$ on the averaged cross section uncertainties.

Next, we examine the correlation between the resonance widths. In capture measurements the capture kernel,

$$
A_{\gamma}=\frac{g \Gamma_{n} \Gamma_{\gamma}}{\Gamma}
$$

shows that there is negative correlation between $\Gamma_{n}$ and $\Gamma_{\gamma}$. This correlation may or may not be strong, depending on the values of the resonance widths involved. Thus, if either $\Gamma_{n} / \Gamma$ or $\Gamma_{\gamma} / \Gamma$ is close to the unity, the correlation is weak. If, however, these ratios are approximately equal, then the correlation between $\Gamma_{n}$ and $\Gamma_{\gamma}$ will be strong. The corresponding expression for the cross section uncertainty reads

$$
\left(\Delta \sigma_{\gamma}\right)^{2}=\left(\frac{\partial \sigma_{\gamma}}{\partial \Gamma_{n}} \Delta \Gamma_{n}\right)^{2}+2 \frac{\partial \sigma_{\gamma}}{\partial \Gamma_{n}}\left\langle\delta \Gamma_{n} \delta \Gamma_{\gamma}\right\rangle \frac{\partial \sigma_{\gamma}}{\partial \Gamma_{\gamma}}+\left(\frac{\partial \sigma_{\gamma}}{\partial \Gamma_{\gamma}} \Delta \Gamma_{\gamma}\right)^{2},
$$

where we again dropped the fission term for simplicity.

The approach described here to calculate the correlation term between the resonance widths applies the generalized least squares method from the Bayesian theorem [12]. The initial values of $\Gamma_{n}, \Gamma_{\gamma}, A_{\gamma}$ as well as their uncertainties, $\Delta \Gamma_{n}, \Delta \Gamma_{\gamma}$ and $\Delta A_{\gamma}$, can be taken from the Atlas of Neutron Resonances. The following relations hold for the prior covariance matrix of the resonance widths, $\Psi$, and the posterior matrix, $\tilde{\Psi}$,

$$
\begin{aligned}
\tilde{\chi} & =\chi+\Psi S^{\mathrm{T}} V[A-A(\chi)] \\
\tilde{\Psi} & =\Psi-\Psi S^{\mathrm{T}} V S \Psi,
\end{aligned}
$$




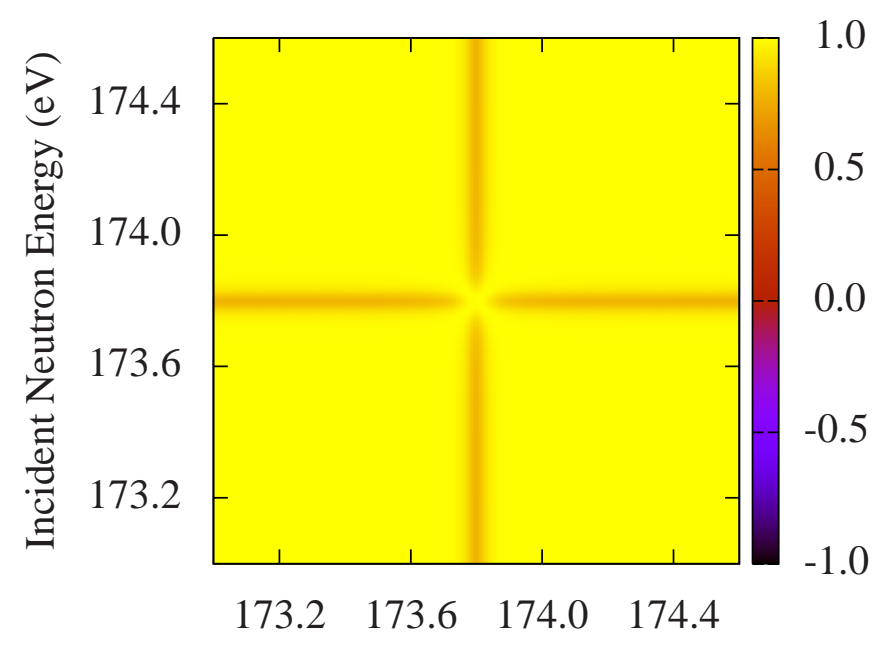

Incident Neutron Energy (eV)

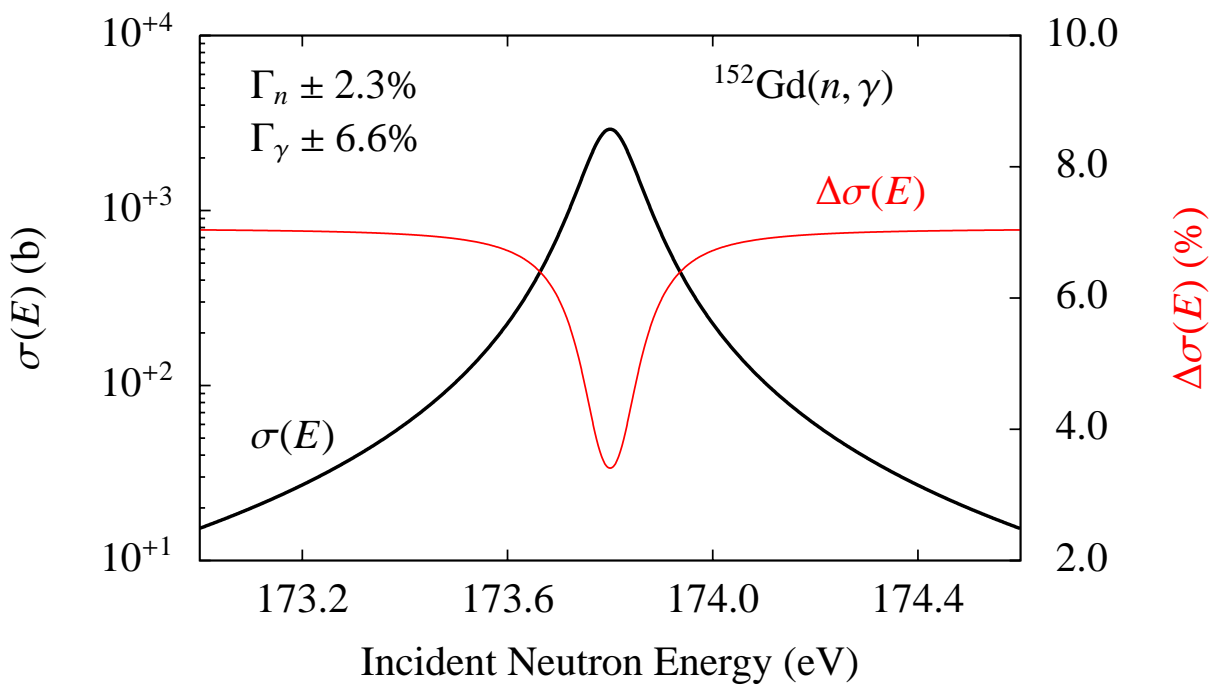

Figure 3.4: Top: The ${ }^{152} \operatorname{Gd}(n, \gamma)$ cross section correlations due to uncorrelated $\Gamma_{n}$ and $\Gamma_{\gamma}$ for the single $173.8 \mathrm{eV}$ resonance. Bottom: The same for relative cross section uncertainties. 

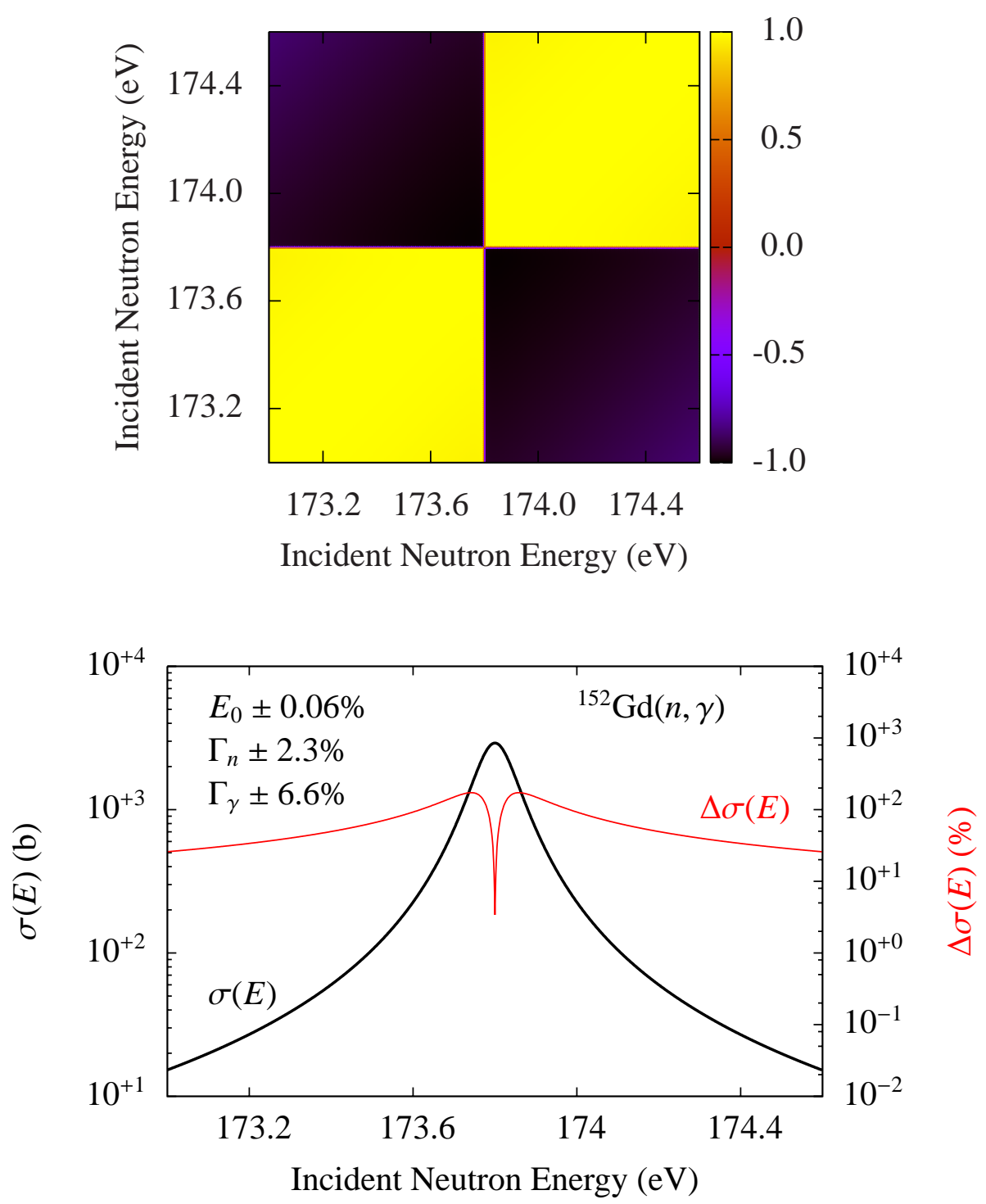

Figure 3.5: Top: The ${ }^{152} \mathrm{Gd}(n, \gamma)$ cross section correlations due to uncorrelated $E_{0}, \Gamma_{n}$ and $\Gamma_{\gamma}$ for the single $173.8 \mathrm{eV}$ resonance. Bottom: The same for relative cross section uncertainties. 
where $V=\left(S \Psi S^{\mathrm{T}}+(\Delta A)^{2}\right)^{-1}$. The vector $A(\chi)$ represents the capture kernel calculated for the set of parameters $\chi \equiv\left\{\Gamma_{n}, \Gamma_{\gamma}\right\}$. The quantity $A \equiv A_{\gamma}$ is the experimental value of the capture kernel with related variance $\left(\Delta A_{\gamma}\right)^{2}$, while $S$ is the sensitivity matrix and $S^{\mathrm{T}}$ is its transpose given by

$$
S^{\mathrm{T}} \equiv\left(\frac{\partial A_{\gamma}}{\partial \Gamma_{n}}, \frac{\partial A_{\gamma}}{\partial \Gamma_{\gamma}}\right) .
$$

The covariance matrix for the resonance parameters is given as

$$
\Psi=\left(\begin{array}{cc}
\left(\Delta \Gamma_{n}\right)^{2} & \left\langle\delta \Gamma_{n} \delta \Gamma_{\gamma}\right\rangle \\
\left\langle\delta \Gamma_{\gamma} \delta \Gamma_{n}\right\rangle & \left(\Delta \Gamma_{\gamma}\right)^{2}
\end{array}\right) .
$$

We introduce the shortened notation for the correlation term between $\Gamma_{n}$ and $\Gamma_{\gamma}$

$$
C=\frac{\left\langle\delta \Gamma_{n} \delta \Gamma_{\gamma}\right\rangle}{\Delta \Gamma_{n} \Delta \Gamma_{\gamma}} .
$$

The upper line of Eq.(3.10) represents the update of the $\Gamma_{n}$ and $\Gamma_{\gamma}$ parameters, while the lower line defines the covariance calculation for these parameters. In the prior matrix $\Psi$, the correlation term $C$ is assumed to be equal to zero. Then, the calculation is iterated by replacing $\Psi$ with the calculated $\tilde{\Psi}$ until convergence is achieved.

We illustrate impact of the $\Gamma_{n}-\Gamma_{\gamma}$ correlations on capture cross section uncertainties in Fig. 3.6. We choose ${ }^{152} \mathrm{Gd}(n, \gamma)$ reaction in the vicinity of the resonance at $173.8 \mathrm{eV}$ and show the range of uncertainties when the correlation coefficient $C$ varies between -0.1 and -0.9 . One notes that low correlations result in higher uncertainties at both wings of the resonance while the opposite is true for the peak zone. The change in the cross section uncertainty can reach about $50 \%$ between physical limits of $C$ (-1 to 0 ) but is less than $30 \%$ in the peak zone. Typical scale of the $\Gamma_{n}-\Gamma_{\gamma}$ correlation is shown in Table 3.2, in which we reproduce experimental values of $C$ for several s-wave resonances in ${ }^{152} \mathrm{Gd}+n$ as reported in Ref. [18]. Generally, there is a strong negative correlation if $\Gamma_{n}$ and $\Gamma_{\gamma}$ are comparable and it weakens if one of the widths becomes much larger.

\subsection{Averaged values}

Users of neutron cross section data are primarily interested in the group-averaged cross sections and their uncertainties. Therefore, it is of practical interest to examine the impact of the covariances on the cross sections that are averaged over 
Table 3.2: The resonance parameters and capture kernels of selected s-wave resonances for ${ }^{152} \mathrm{Gd}+\mathrm{n}$ [10]. The correlation terms, $C$, between $\Gamma_{n}$ and $\Gamma_{\gamma}$ were taken from Ref. [18]. For all resonances $g=1$.

\begin{tabular}{cccccc}
\hline$E_{0}(\mathrm{eV})$ & $g \Gamma_{n}(\mathrm{meV})$ & $\Gamma_{\gamma}(\mathrm{meV})$ & $A_{\gamma}(\mathrm{meV})$ & $C$ & Comment \\
\hline 173.8 & $86 \pm 2$ & $30 \pm 2$ & $22.3 \pm 0.3$ & -0.91 & \\
185.7 & $84 \pm 2$ & $53 \pm 5$ & $32.3 \pm 0.5$ & -0.95 & \\
203.1 & $97 \pm 2$ & $59 \pm 3$ & $36.6 \pm 0.4$ & -0.95 & \\
223.3 & $301 \pm 12$ & $64 \pm 3$ & $52.9 \pm 0.6$ & -0.75 & $\Gamma_{n}>>\Gamma_{\gamma}$ \\
231.4 & $46 \pm 4$ & $62 \pm 8$ & $26.4 \pm 0.9$ & -0.98 & \\
1678.4 & $999 \pm 116$ & $69 \pm 7$ & $64.6 \pm 2.3$ & -0.60 & $\Gamma_{n}>>\Gamma_{\gamma}$ \\
\hline
\end{tabular}

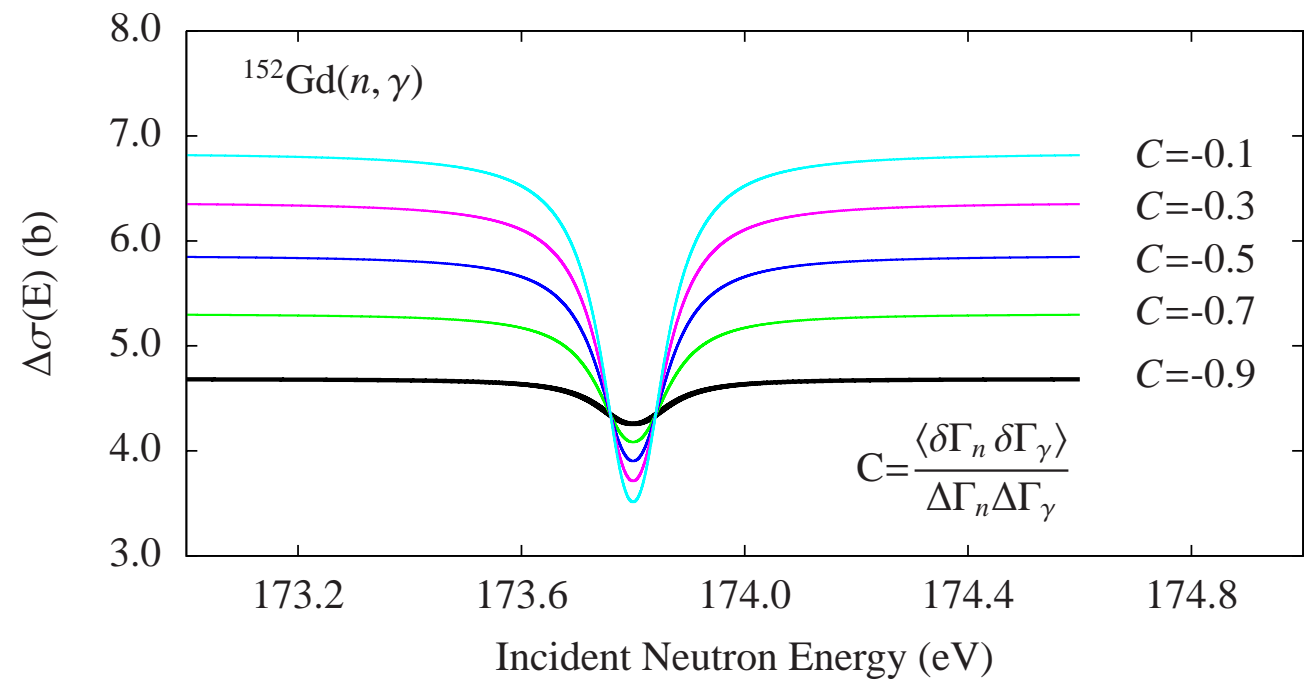

Figure 3.6: $T$ The ${ }^{152} \mathrm{Gd}(\mathrm{n}, \gamma)$ relative cross section uncertainties for the single $173.8 \mathrm{eV}$ resonance illustrating the impact of the correlation between $\Gamma_{n}$ and $\Gamma_{\gamma}$. 
a broader energy interval. The capture cross section averaged over the energy interval $\Delta E$ around the energy $E_{0}$ can be calculated as

$$
\overline{\sigma_{\gamma}}=\frac{1}{\Delta E} \sum_{i} \sigma_{\gamma}\left(E_{i}\right) \Delta e,
$$

where $\Delta e$ is a sufficiently small energy step. Then, the averaged cross section uncertainty is

$$
\overline{\Delta \sigma_{\gamma}}=\frac{\Delta e}{\Delta E} \sqrt{\sum_{i, j}\left\langle\delta \sigma_{\gamma}\left(E_{i}\right) \delta \sigma_{\gamma}\left(E_{j}\right)\right\rangle} .
$$

It should be pointed out that typical widths of energy bins over which the averaging is done is much larger than the width of a single resonance. Thus, in our sample case that we choose to illustrate our results, ${ }^{152} \mathrm{Gd}(n, \gamma)$, the $173.8 \mathrm{eV}$ resonance falls in the group-energy interval that is orders of magnitude larger than the resonance width $\Gamma_{\gamma}=0.03 \mathrm{eV}$. Indeed, in the 44-group structure used for nuclear criticality safety applications the relevant energy group has width orders of magnitude larger. In the 15-group structure, used in some advanced reactor systems studies, the relevant energy group spans the energy range from $22.6 \mathrm{eV}$ to $454 \mathrm{eV}$, implying the bin widths more than $400 \mathrm{eV}$. The energy interval over which the cross section uncertainty is displayed in the above example, see Figs. 3.1-3.6 is less than $1 \mathrm{eV}$. This energy interval is sufficiently broad for our purposes, yet still pretty small when compared to the energy interval of any relevant group structure used in practice.

One important comment is in place. In calculating average quantities the role of correlations become important as can be seen in Eq.(3.15). As a consequence, averaged uncertainties are lower, sometimes considerably lower, than those intuitively expected considering purely diagonal terms.

Considering the anti-correlation caused by $\Delta E_{0}$, it is clear that impact of $\Delta E_{0}$ on the averaged cross section uncertainty is negligible. On the contrary, $\Delta \Gamma_{n}$ and $\Delta \Gamma_{\gamma}$ are important in view of the cross section uncertainties since the related cross section correlation matrix is positive and fairly uniform. Therefore there is no cancellation that eliminates the effect of $\Delta E_{0}$. The impact of the correlation between $\Gamma_{n}$ and $\Gamma_{\gamma}$ may be significant and reduces the average cross section uncertainty for negative $C$. 


\section{Chapter 4}

\section{Cross section covariances for multiple resonances}

The previous analysis can be extended to a more realistic case with many resonances. We will discuss the cross section uncertainties and then proceed with the correlations.

\subsection{Cross section uncertainties}

Using Eq. (3.4), the cross section uncertainty for the multi-resonance case can be worked out fairly easily. Two cases will be discussed, first we would assume uncorrelated resonance parameters, afterwards we will consider correlation between $\Gamma_{n}$ and $\Gamma_{\gamma}$. For the uncorrelated resonance parameters one has

$$
\left(\Delta \sigma_{\gamma}\right)^{2}=\sum_{r}\left[\left(\frac{\partial \sigma_{\gamma}}{\partial E_{0 r}} \Delta E_{0 r}\right)^{2}+\left(\frac{\partial \sigma_{\gamma}}{\partial \Gamma_{n r}} \Delta \Gamma_{n r}\right)^{2}+\left(\frac{\partial \sigma_{\gamma}}{\partial \Gamma_{\gamma r}} \Delta \Gamma_{\gamma r}\right)^{2}\right],
$$

where $r$ denotes the individual resonances. Following Eqs. (3.5) and (3.6) the partial contributions to $(\Delta \sigma)^{2}$ can be readily obtained and, after some rearrangement and dropping subscript $\gamma$, written as

$$
\frac{\partial \sigma}{\partial E_{0 r}} \frac{\Delta E_{0 r}}{\sigma}=\frac{\sigma_{r}}{\sigma}\left(\frac{8 E_{0}\left(E-E_{0 r}\right)}{\Gamma_{r}^{2}+4\left(E-E_{0 r}\right)^{2}}-\frac{1}{2}\right) \frac{\Delta E_{0 r}}{E_{0 r}}
$$

and

$$
\frac{\partial \sigma}{\partial \Gamma_{x r}} \frac{\Delta \Gamma_{x r}}{\sigma}=\frac{\sigma_{r}}{\sigma}\left(1-\frac{2 \Gamma_{x r} \Gamma_{r}}{\Gamma_{r}^{2}+4\left(E-E_{0 i}\right)^{2}}\right) \frac{\Delta \Gamma_{x r}}{\Gamma_{x r}}
$$


where $\sigma_{r}$ is the cross section of the resonance $r$ and $x=n, \gamma$. The ratio $\sigma_{r} / \sigma$ modifies the behavior of the cross section uncertainty far from the resonance energy $E_{0 r}$. If the neutron energy $E$ is close to $E_{0 r}$, then the ratio $\sigma_{r} / \sigma$ is almost equal to unity and Eqs. $(4.2,4.3)$ become similar to Eqs. $(3.5,3.6)$. For the energy $E$ far from $E_{0 r}$, the $\sigma_{r} / \sigma$ becomes small in the presence of another resonance and the effect of the $r^{\text {th }}$ resonance on the cross section uncertainty is also small.

We will discuss two examples, each showing three s-wave resonances. Our first example continues with the case of ${ }^{152} \mathrm{Gd}(n, \gamma)$. We already discussed the $173.8 \mathrm{eV}$ resonance, now we proceed by adding $185.7 \mathrm{eV}$ and $203.1 \mathrm{eV}$ resonances. For these three resonances, the calculated capture cross sections and the calculated relative uncertainties are shown in Fig. 4.1. One can see three broad peaks in the uncertainty curve with narrow dips at the resonance energies. Possible impact of the correlation between $\Gamma_{n}$ and $\Gamma_{\gamma}$ is displayed by the shadowed band that corresponds to the range of values $C=0.0$ and -0.9 .

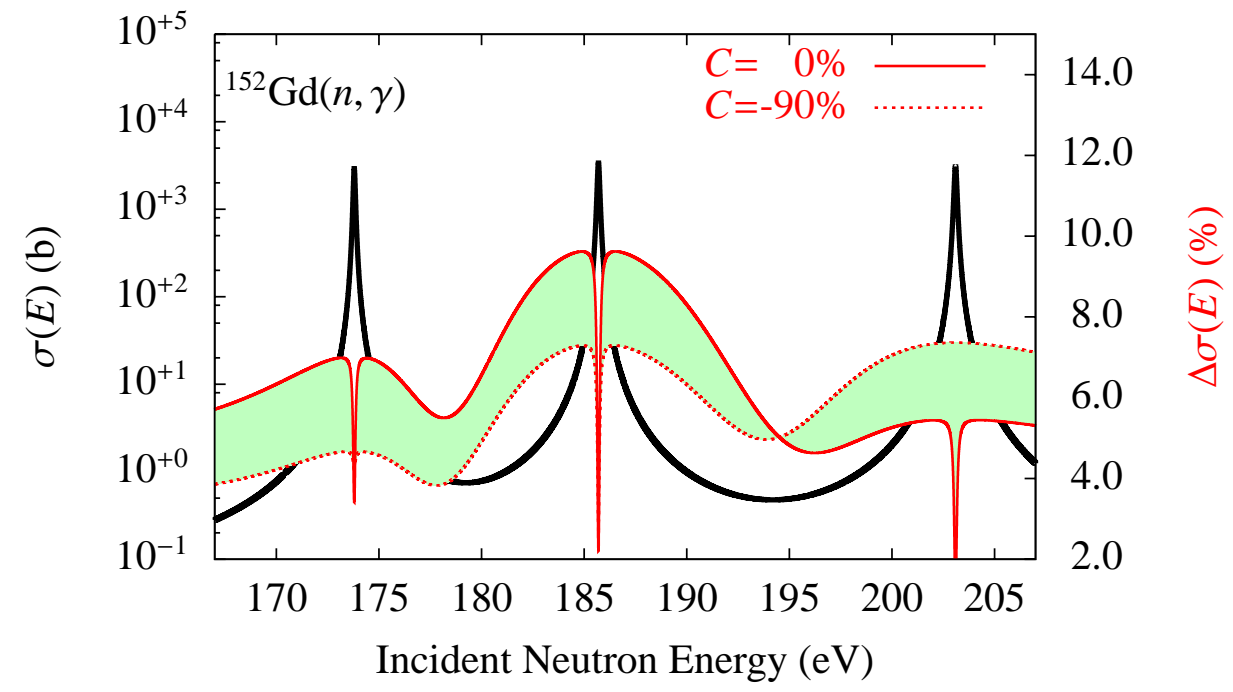

Figure 4.1: The ${ }^{152} \mathrm{Gd}(n, \gamma)$ cross sections and their relative uncertainties for three s-wave resonances, $E_{0}=173.8,185.7$ and $203.1 \mathrm{eV}$. The resonance energy uncertainties, $\Delta E_{0}$, were not considered. The shadowed band illustrates the impact of the $\left(\Gamma_{n}, \Gamma_{\gamma}\right)$ correlation.

Our second example discusses fission. In Fig. 4.3 we show ${ }^{241} \mathrm{Am}(\mathrm{n}, \mathrm{f})$ cross sections and their uncertainties considering three resonances as well as the bound level. The resonance parameters and their uncertainties are given in Table 4.1. The contribution of the bound level to the cross sections is clearly visible. One 


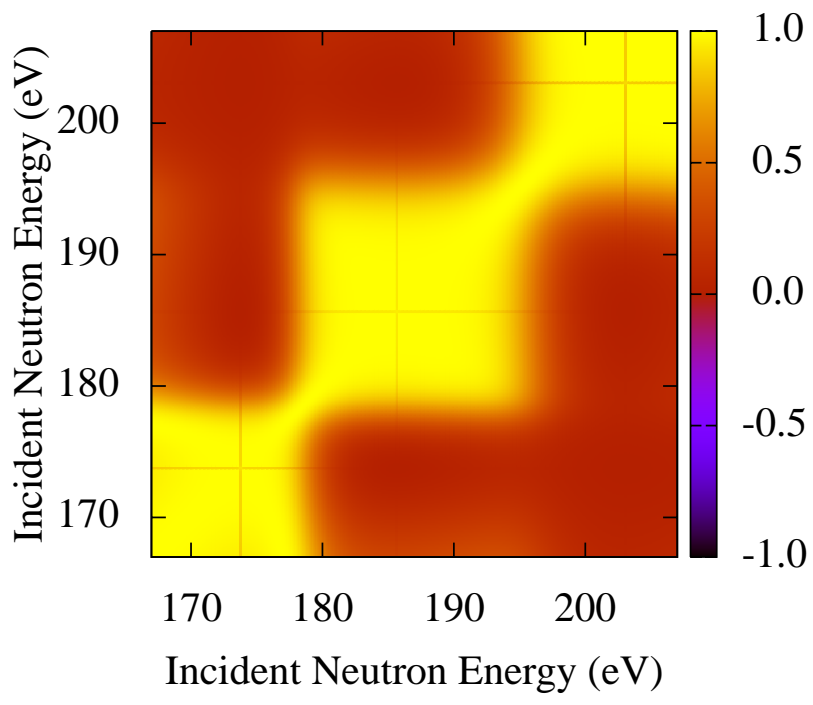

Figure 4.2: Cross section correlation due to uncorrelated $\Gamma_{n}$ and $\Gamma_{\gamma}$ for ${ }^{152} \operatorname{Gd}(n, \gamma)$ for three s-wave resonances, $E_{0}=173.8,185.7$ and $203.1 \mathrm{eV}$.

Table 4.1: The resonance parameters and their uncertainties for three s-wave resonances in ${ }^{241} \mathrm{Am}(\mathrm{n}, \mathrm{f})$ [10], fission kernels $A_{f}$ are not available. Also shown are parameters for the bound state which are considered to be known exactly. Shown in the last column are correlation coefficients, $C$, between $\Gamma_{n}$ and $\Gamma_{f}$.

\begin{tabular}{cccc}
\hline$E_{0}(\mathrm{eV})$ & $2 g \Gamma_{n}(\mathrm{meV})$ & $\Gamma_{\gamma}(\mathrm{meV})$ & $\Gamma_{f}(\mathrm{meV})$ \\
\hline-0.425 & 0.641 & 40 & 0.215 \\
$0.307 \pm 0.002$ & $0.0560 \pm 0.0005$ & $46.8 \pm 0.3$ & $0.29 \pm 0.03$ \\
$0.574 \pm 0.004$ & $0.0923 \pm 0.0020$ & $47.2 \pm 0.3$ & $0.14 \pm 0.02$ \\
$1.268 \pm 0.004$ & $0.3200 \pm 0.0080$ & $48.9 \pm 0.7$ & $0.37 \pm 0.02$ \\
\hline
\end{tabular}




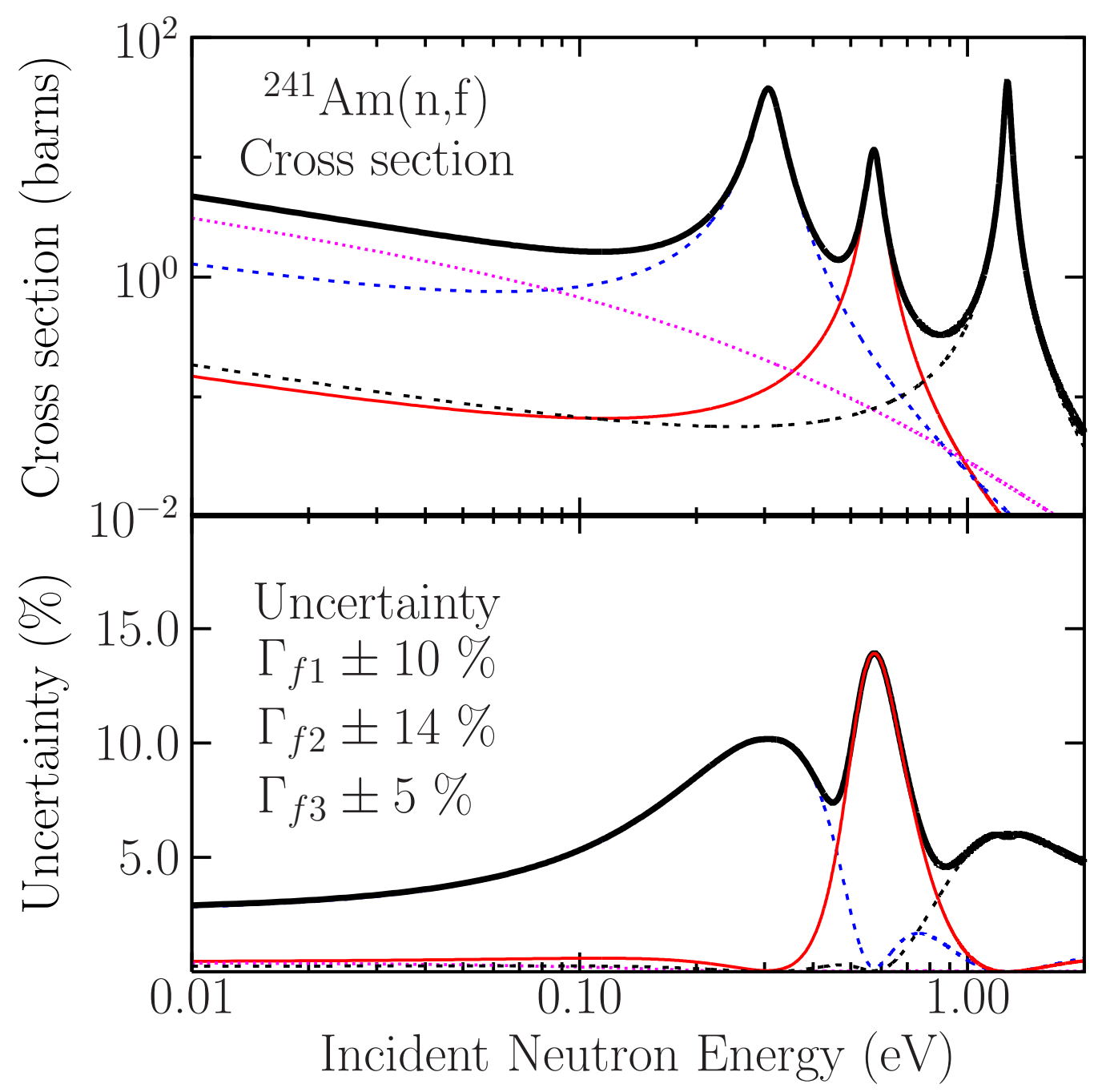

Figure 4.3: The ${ }^{241} \mathrm{Am}(\mathrm{n}, \mathrm{f})$ cross sections and their relative uncertainties for three swave resonances $(0.307,0.574$ and $1.268 \mathrm{eV})$ and the bound level. The resonance energy uncertainties, $\Delta E_{0}$, were not considered. 
can see that there are no local mimima at the resonance energies, in line with our earlier discussion of the single resonances as $\Delta \Gamma_{f} / \Gamma$ is close to zero. Since the resonances are close to each other the local structures are washed out due to the uncertainties of individual resonances.

Table 4.1 shows the resonance parameters for three s-wave resonances in ${ }^{241} \mathrm{Am}(\mathrm{n}, \mathrm{f})$ as well as the bound level and we expect $\left(\Gamma_{n}, \Gamma_{\gamma}\right)$ to be strongly anticorrelated.

\subsection{Cross section correlations}

The energy-energy correlation between capture (fission) cross sections for many resonances can be obtained readily using Eq. (3.7) and performing summation of contributions from single resonances $r$. One has

$$
\frac{\left\langle\delta \sigma(E) \delta \sigma\left(E^{\prime}\right)\right\rangle}{\Delta \sigma(E) \Delta \sigma\left(E^{\prime}\right)}=\sum_{r} \sum_{v} \frac{\partial \sigma(E)}{\partial p_{v r}} \frac{\left(\Delta p_{v r}\right)^{2}}{\Delta \sigma(E) \Delta \sigma\left(E^{\prime}\right)} \frac{\partial \sigma\left(E^{\prime}\right)}{\partial p_{v r}},
$$

where the subscript $v$ denotes different resonance parameters. When discussing correlations one can consider three options, although they may not be fully supported by the data available in the Atlas of Neutron Resonances. These options are:

- Uncorrelated parameters for each individual resonance,

- Correlations between parameters of a single resonance (short range correlation), and

- Correlations between parameters of various resonances (long range correlation).

The first option is illustrated on ${ }^{241} \mathrm{Am}(\mathrm{n}, \mathrm{f})$ reactions in Fig. 4.4. The resonance parameters and their uncertainties, given in Table 4.1, are treated as uncorrelated. Strong and localized anti-correlation can be seen close to the resonance energies. For ${ }^{241} \mathrm{Am}(\mathrm{n}, \mathrm{f})$, the cross section uncertainty in the thermal energy region is dominated by the $0.307 \mathrm{eV}$ resonance. Consequently, the thermal cross section and uncertainty are almost fully dominated by the first positive resonance at $0.307 \mathrm{eV}$. The second option could be illustrated by continuing in the above example and including the effect of $\Gamma_{n}$ and $\Gamma_{f}$ correlation. It appears that, when looking on the 


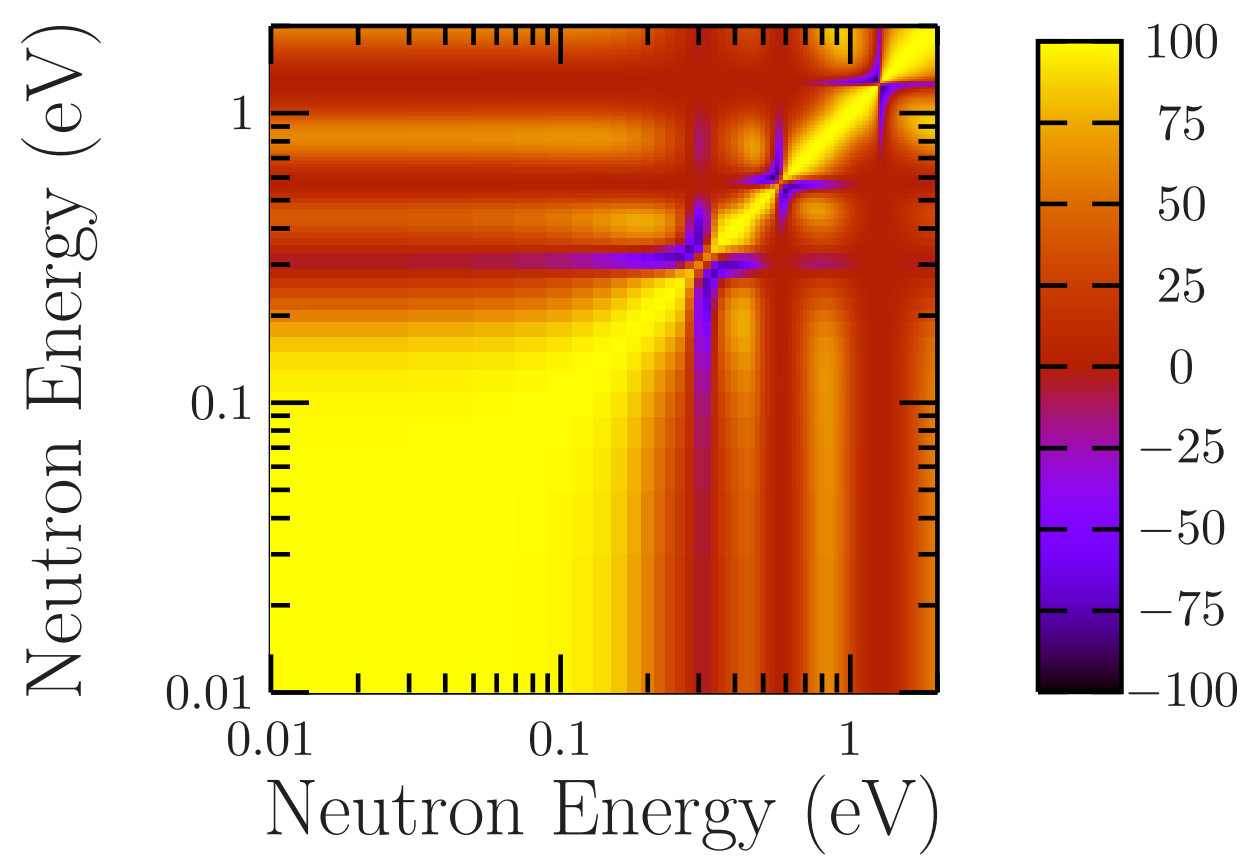

Figure 4.4: Fission cross section correlations for ${ }^{241} \mathrm{Am}(\mathrm{n}, \mathrm{f})$ considering three resonances $(0.307,0.574$ and $1.268 \mathrm{eV})$ and the bound level. The uncertainties of all resonance parameters were assumed to be uncorrelated. 
correlation plot similar to Fig. 4.4, the effects are relatively small and hence not shown here.

The third option takes into account also long-range correlations. Obviously one could consider the resonance energies as they are determined by the neutron flight path, but this effect in practice is very small and can be neglected. Of more interest would be to consider another correlation, indicated by the Atlas of Neutron Resonances, though without any strict guidance. This correlation can be inferred from the fact that often the radiative widths are assumed to be constant. In this case, the radiative widths of all resonances should be strongly correlated. Such correlations can be only estimated using ad hoc assumptions as no guidance is given in the Atlas of Neutron Resonances and we are not attempting to do so here.

\subsection{Averaged values}

As already mentioned the users require multi-group cross sections. The reason is that large simulation codes are not designed for point-wise cross sections that are far too detailed, rather one needs suitably averaged values, the multi-group cross sections. To this end, the processing codes such as PUFF [15] and ERRORJ [16] and NJOY [17]should be employed.

From the above discussion it is clear that the two possible ways how to obtain multi-group cross section uncertainties in the resonance region should be equivalent. If one choses to produce MF32 covariances, then PUFF or ERRORJ should be used to obtain multi-group cross section covariances from covariances of resonances parameters. If, alternatively, one chose to produce MF33 covariances, then either of the above codes can be used to obtain multi-group cross section covariances. We are not resorting to show this on any single case as such an example might not be considered as sufficiently general and it is beyond the scope of this report to go to extensive analysis of this point.

In practice, MF32 is more straightforward and provides more flexibility. Hence its use, unless prohibited by huge size of the file, such as in the case of ${ }^{235} \mathrm{U}$, is preferable. 


\section{Chapter 5}

\section{Conclusions}

The cross section uncertainties and correlations for neutron capture and fission in the resolved resonance region were examined. Our goal was to make maximum use of the information available in the Atlas of Neutron Resonances. We used the MLBW formalism that allowed analytical solutions, complemented with the numerical calculations whenever necessary.

We studied the impact of the resonance parameter $\left(E_{0}, \Gamma_{\gamma}, \Gamma_{n}\right)$ uncertainties and examined the possibility to introduce resonance parameter correlations by utilizing the capture kernel $\left(A_{\gamma}\right)$. We have shown that the uncertainties of the resonance energies, $\Delta E_{0}$, can be neglected in the averaged cross sections. The uncertainties of the other resonance parameters should be taken into account. This is also true for the correlations between $\Gamma_{n}$ and $\Gamma_{\gamma}$ in cases where these widths have comparable values.

The use of the resonance parameter covariances, file MF32, is a logical step forward in developing our covariance methodology in the neutron resolved resonance region. So far, we have been using the cross section covariance representation, file MF33. These two ways are equivalent in the sense of providing the same multi-group values, but the use of MF32 is more straightforward and more flexible and it should be given the preference.

We conclude that the Atlas of Neutron Resonances contains the wealth of information that can be effectively utilized in the evaluation of neutron cross section covariances in the resolved energy region. 


\section{List of Figures}

3.1 The ${ }^{152} \mathrm{Gd}(n, \gamma)$ cross sections for the single resonance $E_{0}=173.8 \mathrm{eV}$ (left scale) and their relative uncertainties due to the resonance energy uncertainty $0.06 \%$ (right scale). . . . . . . . . . 8

3.2 The ${ }^{152} \mathrm{Gd}(n, \gamma)$ cross sections for the single $173.8 \mathrm{eV}$ resonance (left scale) and their relative uncertainties due to the neutron width $\Gamma_{n}=86 \mathrm{meV}_{ \pm 2} .3 \%$ (right scale). . . . . . . . . . . . 9

3.3 The ${ }^{152} \mathrm{Gd}(n, \gamma)$ cross sections for the single $173.8 \mathrm{eV}$ resonance (left scale) and their relative uncertainties due to the radiative width $\Gamma_{\gamma}=30 \mathrm{meV} \pm 6.6 \%$ (right scale). . . . . . . . . . 9

3.4 Top: The ${ }^{152} \mathrm{Gd}(n, \gamma)$ cross section correlations due to uncorrelated $\Gamma_{n}$ and $\Gamma_{\gamma}$ for the single $173.8 \mathrm{eV}$ resonance. Bottom: The same for relative cross section uncertainties. . . . . . . . . . 11

3.5 Top: The ${ }^{152} \mathrm{Gd}(n, \gamma)$ cross section correlations due to uncorrelated $E_{0}, \Gamma_{n}$ and $\Gamma_{\gamma}$ for the single $173.8 \mathrm{eV}$ resonance. Bottom: The same for relative cross section uncertainties. . . . . . . . . 12

3.6 The ${ }^{152} \mathrm{Gd}(\mathrm{n}, \gamma)$ relative cross section uncertainties for the single $173.8 \mathrm{eV}$ resonance illustrating the impact of the correlation between $\Gamma_{n}$ and $\Gamma_{\gamma} \ldots \ldots \ldots \ldots \ldots \ldots$

4.1 The ${ }^{152} \operatorname{Gd}(n, \gamma)$ cross sections and their relative uncertainties for three s-wave resonances, $E_{0}=173.8,185.7$ and $203.1 \mathrm{eV}$. The resonance energy uncertainties, $\Delta E_{0}$, were not considered. The shadowed band illustrates the impact of the $\left(\Gamma_{n}, \Gamma_{\gamma}\right)$ correlation. . . . . 17

4.2 Cross section correlation due to uncorrelated $\Gamma_{n}$ and $\Gamma_{\gamma}$ for ${ }^{152} \operatorname{Gd}(n, \gamma)$ for three s-wave resonances, $E_{0}=173.8,185.7$ and $203.1 \mathrm{eV}$. . . 18 
4.3 The ${ }^{241} \mathrm{Am}(\mathrm{n}, \mathrm{f})$ cross sections and their relative uncertainties for three s-wave resonances $(0.307,0.574$ and $1.268 \mathrm{eV})$ and the bound level. The resonance energy uncertainties, $\Delta E_{0}$, were not considered. . . . . . . . . . . . . . . . . . 19

4.4 Fission cross section correlations for ${ }^{241} \mathrm{Am}(\mathrm{n}, \mathrm{f})$ considering three resonances $(0.307,0.574$ and $1.268 \mathrm{eV})$ and the bound level. The uncertainties of all resonance parameters were assumed to be uncorrelated. . . . . . . . . . . . . . 21 


\section{List of Tables}

3.1 The resonance parameters and their uncertainties for $E_{0}=173.8$ eV s-wave resonance in ${ }^{152} \mathrm{Gd}+n[10] . \ldots . . . . . . . .77$

3.2 The resonance parameters and capture kernels of selected s-wave resonances for ${ }^{152} \mathrm{Gd}+\mathrm{n}$ [10]. The correlation terms, $C$, between $\Gamma_{n}$ and $\Gamma_{\gamma}$ were taken from Ref. [18]. For all resonances $g=1$. . . 14

4.1 The resonance parameters and their uncertainties for three s-wave resonances in ${ }^{241} \mathrm{Am}(\mathrm{n}, \mathrm{f})$ [10], fission kernels $A_{f}$ are not available. Also shown are parameters for the bound state which are considered to be known exactly. Shown in the last column are correlation coefficients, $C$, between $\Gamma_{n}$ and $\Gamma_{f} \ldots \ldots \ldots \ldots$ 


\section{Acknowledgments}

The authors wish to thank Toshihiko Kawano, LANL for the stimulation and encouragement. We gratefully acknowledge useful discussions with G. Chiba, Japan Atomic Energy Agency (JAEA). This manuscript has been authored by Brookhaven Science Associates, LLC, under contract DELACO2-98CH10886 with the U.S. Department of Energy. 


\section{Bibliography}

[1] M. Salvatores, G. Aliberti, G. Palmiotti, D. Rochman, P. Obložinský, M. Herman, P. Talou, T. Kawano, L. Leal, A. Koning, I. Kodeli, "Nuclear Data Needs for Advanced Reactor Systems. A NEA Nuclear Science Committee Initiative", Proceedings of the International Conference on Nuclear Data for Science and Technology, Nice, France, April 22-27, 2007, to be published.

[2] G. Aliberti, G. Palmiotti, M. Salvatores, T. K. Kim, T. A. Taiwo, M. Anitescu, I. Kodeli, E. Sartori, J. C. Boscp and J. Tommasi, Annals of Nuclear Energy 33 (2006) 700.

[3] M.B. Chadwick, P. Obložinský, M. Herman et al., "ENDF/B-VII.0: Next Generation of Evaluated Nuclear Data Library for Nuclear Science and Technology", Nuclear Data Sheets 107 (2006) 2931.

[4] N.M. Larson, "Updated Users' Guide for SAMMY: Multilevel R-Matrix Fits to Neutron Data Using Bayes' Equations", Report ENDF-364 and ORNL/TM-9179/R6, Oak Ridge National Laboratory, Oak Ridge, TN (May 2003). Also report ORNL/TM-9179/R7 (2006).

[5] N.M. Larson, "Use of Covariance Matrices in SAMMY", Workshop on Nuclear Data Evaluation for Reactor Applications, WONDER 2006, 9-11 October 2006, Château de Cadarache, Saint-Paul-lez-Durance, France, unpublished.

[6] M.C. Moxon and J.B. Brisland, "REFIT, a least square fitting program for resonance analysis of neutron transmission and capture data", Technical Report AEA-INTEC-0630, AEA Technology (1991).

[7] A. Borella, G. Aerts, F. Gunsing, M. Moxon, P. Schillebeeckx and R. Wynants, Nucl. Inst. Meth. A 577 (2007) 626. 
[8] P. Schillebeeckx, private communication, July 2007.

[9] T. Kawano, L.C. Leal et al, "Covariance matrix evaluation and processing in the resolved/unresolved resonance region", Report WPEC-Subgroup 20, NEA/WPEC-20, OECD, Paris, 2006.

[10] S.F. Mughabghab, "Atlas of Neutron Resonances: Thermal Cross Sections and Resonance Parameters", Elsevier Publisher, Amsterdam, 2006.

[11] M. Herman, R. Capote, B. Carlson et al, "EMPIRE: Nuclear Reaction Model Code System for Data Evaluation”, Nuclear Data Sheets 108 (2007) 2655.

[12] T. Kawano and K. Shibata, "Covariance Evaluation System", in Japanese, JAERI Report JAERI-Data/Code 97-037 (1997).

[13] D. Rochman, M. Herman, P. Obložinský, S. Mughabghab, "Preliminary Cross Section and Nubar Covariances for WPEC Subgroup 26 (Nuclear Data Needs for Advanced Reactor Systems)", Internal Report BNL-77407-2007IR (2007).

[14] M. Herman (editor), "ENDF-6 Formats Manual: Data Formats and Procedures for the Evaluated Nuclear Data File ENDF/B-VI and ENDF/B-VII", Tech. Report BNL-NCS-44945-05-Rev, Document ENDF-102, Brookhaven National Laboratory, 2005.

[15] D. Wiarda and M.E. Dunn, "PUFF-IV: A Code for Processing ENDF Uncertainty Data into Multigroup Covariance Matrices", Report ORNL/TM2006/147 (2006).

[16] G. Chiba, "ERRORJ-2.2 Manual Report on JENDL-3.2 Covariance Matrices", Report JAERI-Research 2003-001 (2003).

[17] R.E. McFarlane and D.W. Muir, “The NJOY Nuclear Data Processing System, Version 91", Los Alamos National Laboratory, Report LA-12740-M (1994), and more recent updates.

[18] R.L. Macklin, "Neutron Capture Resonances of ${ }^{152}$ Gd and ${ }^{154}$ Gd”, Nucl. Sci. Eng. 95 (1987) 304. 